\title{
Benzodiazepine use and risk of incident dementia or cognitive decline: prospective population based study
}

\author{
Shelly L Gray, ${ }^{1}$ Sascha Dublin, ${ }^{2}$ Onchee Yu, ${ }^{2}$ Rod Walker, ${ }^{2}$ Melissa Anderson, ${ }^{2}$ Rebecca A Hubbard, ${ }^{3}$ \\ Paul K Crane, ${ }^{4}$ Eric B Larson²
}

1School of Pharmacy, University of Washington, Seattle, WA

98195-7630, US

${ }^{2}$ Group Health Research Institute, Group Health Cooperative, Seattle, WA, US

${ }^{3}$ Department of Biostatistics and Epidemiology, Perelman School of Medicine, University of Pennsylvania, Pennsylvania, PA, US

4 University of Washington Department of Medicine, Division of General Internal Medicine, Seattle, WA, US

Correspondence to: S Gray slgray@u.washington.edu Cite this as: BMJ 2016;352:i90 http://dx.doi.org/10.1136/bmj.i90

\section{ABSTRACT}

OBJECTIVE

To determine whether higher cumulative use of benzodiazepines is associated with a higher risk of dementia or more rapid cognitive decline.

DESIGN

Prospective population based cohort.

SETTING

Integrated healthcare delivery system, Seattle, Washington.

PARTICIPANTS

3434 participants aged $\geq 65$ without dementia at study entry. There were two rounds of recruitment (1994-96 and 2000-03) followed by continuous enrollment beginning in 2004.

\section{MAIN OUTCOMES MEASURES}

The cognitive abilities screening instrument (CASI) was administered every two years to screen for dementia and was used to examine cognitive trajectory. Incident dementia and Alzheimer's disease were determined with standard diagnostic criteria. Benzodiazepine exposure was defined from computerized pharmacy data and consisted of the total standardized daily doses (TSDDs) dispensed over a 10 year period (a rolling window that moved forward in time during follow-up). The most recent year was excluded because of possible use for prodromal symptoms. Multivariable Cox proportional hazard models were used to examine time varying use of benzodiazepine and dementia risk. Analyses of cognitive trajectory used linear regression models with generalized estimating equations.

RESULTS

Over a mean follow-up of 7.3 years, 797 participants (23.2\%) developed dementia, of whom 637 developed Alzheimer's disease. For dementia, the adjusted hazard ratios associated with cumulative benzodiazepine use compared with non-use were 1.25 (95\% confidence interval 1.03 to 1.51 ) for $1-30$ TSDDs; 1.31 (1.00 to 1.71) for 31-120 TSDDs; and 1.07 (0.82 to 1.39)

\section{WHAT IS ALREADY KNOWN ON THIS TOPIC}

Benzodiazepine use is common among older adults to manage sleep, anxiety, and depressive disorders

Studies suggest that benzodiazepine use could be associated with an increased risk of dementia, though whether this association is causal remains uncertain

\section{WHAT THIS STUDY ADDS}

The highest level of benzodiazepine use was not associated with incident dementia This study is the first to combine computerized pharmacy data to characterize cumulative benzodiazepine use over a long period (10 years) with rigorous research based standards to diagnose dementia and Alzheimer's disease for $\geq 121$ TSDDs. Results were similar for Alzheimer's disease. Higher benzodiazepine use was not associated with more rapid cognitive decline.

\section{CONCLUSION}

The risk of dementia is slightly higher in people with minimal exposure to benzodiazepines but not with the highest level of exposure. These results do not support a causal association between benzodiazepine use and dementia.

\section{Introduction}

Benzodiazepines are widely prescribed to treat insomnia and anxiety, with about $9-12 \%$ of older adults in the United States reporting use. ${ }^{12}$ These drugs are associated with many deleterious effects, including falls, fractures, traffic incidents, and delirium. ${ }^{13}$ Because of these risks benzodiazepines are not recommended for treatment of insomnia, agitation, or delirium in older adults, and it is recommended that use, if any, be short term. ${ }^{4}$ Nonetheless, benzodiazepine use increases with age, and older adults are more likely to use these drugs long term. ${ }^{1}$

Single dose studies have shown that benzodiazepines impair aspects of cognition (such as memory and attention). ${ }^{5}$ It remains uncertain whether long term use is associated with global cognitive decline. Some well conducted studies suggest that long term use does not increase the risk of cognitive decline, but results are conflicting. ${ }^{67}$ Considerable attention has focused on the potential relation between benzodiazepines and increased risk of dementia. ${ }^{8-14}$ Examination of this relation is challenging because dementia can be preceded by symptoms such as insomnia, anxiety, and depression, ${ }^{15-17}$ which are often treated with benzodiazepines. Observational studies must use appropriate design strategies to account for benzodiazepines used to treat early symptoms of dementia to avoid bias from reverse causation. We are aware of three studies that intentionally considered the prodromal phase and potential for reverse causation. Two of these reported an increased risk of dementia with benzodiazepine use, ${ }^{89}$ while the other did not. ${ }^{14}$ These studies had limitations including lack of information about duration and dose of benzodiazepine treatment ${ }^{8}$ and the reliance on administrative data to identify people with Alzheimer's disease. ${ }^{914}$ No studies have been conducted in the US, where patterns of benzodiazepine use differ from other countries. Given the enormous public health implications, we need a better understanding of the potential cognitive risks of cumulative benzodiazepine use.

We used data from a prospective cohort study with research quality diagnoses of dementia and computerized pharmacy data to evaluate the association 
between cumulative benzodiazepine use and the risk for dementia and cognitive decline. We hypothesized that higher cumulative use would be associated with increased risk.

\section{Methods}

Design, study setting, and participants

The Adult Changes in Thought study is a population based prospective cohort study conducted within Group Health, an integrated healthcare delivery system in the north west US. Study procedures have been reported elsewhere. ${ }^{18}$ Briefly, participants aged $\geq 65$ without dementia were randomly sampled from Group Health members in the Seattle area. The original cohort of 2581 people was enrolled in 1994-96 and 811 additional participants in 2000-03. In 2004 the study began continuous enrollment to replace those who developed dementia, died, or dropped out. Participants were assessed at study entry and biennially thereafter to evaluate cognitive function and collect demographic characteristics, medical history, health behaviors, and health status. Our analyses were limited to participants who had at least 10 years of prior membership of Group Health at enrollment to ensure adequate data on long term exposure to medication (fig 1). For the analyses of dementia, we required participants to have at least one follow-up visit. For the analyses of cognitive trajectory, we included all participants who had a valid cognitive score at baseline. Because we were interested in whether benzodiazepine use was associated with a more rapid cognitive decline only in participants who had not yet had a diagnosis of dementia, we excluded from these analyses any visits made after the date of onset of dementia.

\section{Cognitive outcomes}

Identification of dementia and Alzheimer's disease We used the cognitive abilities screening instrument (CASI) to screen for dementia at study entry and at each biennial study visit. ${ }^{19}$ Scores range from 0 to 100 , with higher scores indicating better performance. Participants with scores of $\leq 85$ underwent a standardized diagnostic evaluation for dementia, including a physical and neurological examination and neuropsycholog-

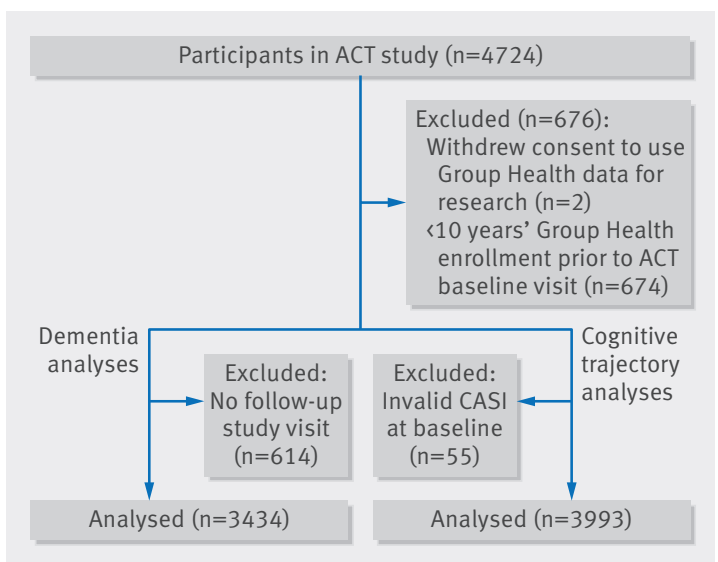

Fig 1 | Sample for analyses of dementia and cognitive trajectory in Adult Changes in Thought (ACT) study ical testing. The results, along with clinical data from participants' medical records, were then reviewed in a multidisciplinary consensus conference including the examining physician, a neuropsychologist, another study physician, and the study nurse. The diagnoses of dementia and Alzheimer's disease were made with standard research criteria. ${ }^{2021}$ The date of onset of dementia was assigned as the midpoint between the study visit that triggered the evaluation of dementia and the preceding visit. Participants with new onset dementia underwent at least one follow-up examination to confirm the diagnosis.

\section{Cognitive trajectory}

We used the CASI score for our primary analyses of cognitive trajectory. A feature of this score is that the distribution of item difficulty is not uniform across levels of cognitive ability. For example, there are few hard questions that would be appropriate for those with no cognitive impairment. Because of this, there is a non-linear relation between the score and underlying cognitive ability, resulting in imprecision at the higher end of the scale. ${ }^{22}$ We therefore performed secondary analyses after applying item response theory (IRT) methods to generate CASI-IRT scores, which have linear scaling properties (Parscale, Scientific Software International, Chicago, IL). ${ }^{23}$ This deals with the relative insensitivity of the CASI to detect cognitive decline in people with high cognitive functioning.

\section{Use of benzodiazepines}

The exposure included benzodiazepines and non-benzodiazepine hypnotics that bind to the gamma-aminobutyric acid (GABA) receptor, such as zolpidem, zaleplon, and eszopiclone. Few participants, however, used non-benzodiazepine hypnotics. Drug use was ascertained from Group Health's computerized pharmacy data and included drug name, strength, route of administration, date dispensed, and amount dispensed.

Previous studies have not delineated what pattern of benzodiazepine exposure might be important for increasing risk of dementia (for example, long term sustained use versus several episodes of periodic use). We hypothesized that cumulative drug exposure, particularly heavier exposure that might accumulate over a long time period (via either intermittent or sustained use), was the most plausible causal mechanism by which use might increase the risk of dementia. We therefore selected a 10 year window based on this hypothesis and on methodologic and practical considerations.

To create our exposure measures, we first calculated the total benzodiazepine dose for each prescription by multiplying the drug strength and the number of tablets dispensed. We then calculated a standardized daily dose (SDD) by dividing the product by the minimum effective dose per day recommended for use in older adults (table 1). ${ }^{24}$ For each participant, we summed the SDDs for all filled prescriptions for benzodiazepines during the 10 year exposure window to create a cumulative total standardized daily dose (TSDD). ${ }^{25-27}$ We constructed a time varying measure defined as the TSDD dispensed over a 


\begin{tabular}{|c|c|}
\hline Benzodiazepine drug & Minimum effective dose (mg) \\
\hline Temazepam & 15 \\
\hline Diazepam & 4 \\
\hline Clonazepam & 0.5 \\
\hline Triazolam & 0.125 \\
\hline Lorazepam & 2 \\
\hline Alprazolam & 0.75 \\
\hline Zolpidem & 5 \\
\hline Flurazepam & 15 \\
\hline Oxazepam & 30 \\
\hline Chlordiazepoxide & 15 \\
\hline Clorazepate & 15 \\
\hline Eszopiclone & 1 \\
\hline Zaleplon & 5 \\
\hline
\end{tabular}

10 year window after excluding dispensings in the most recent year, which could have been for prodromal symptoms of dementia. ${ }^{28}$ Figure 2 shows how exposure windows were defined. At each time point during follow-up, the cumulative exposure for all participants at risk is recalculated by summing all of their benzodiazepine use in the previous 10 years (after exclusion of the most recent year ). We categorized cumulative use as no use, 1-30 TSDDs, 31-120 TSDDs, or $\geq 121$ TSDDs based on the distribution of the exposure and clinically meaningful cutpoints. As examples, a person would reach the highest level of exposure in a 10 year period if he/she took any of the following for a total of 121 days or longer: temazepam $15 \mathrm{mg} /$ day, triazolam $0.125 \mathrm{mg} /$ day, or lorazepam $2 \mathrm{mg} /$ day. This level of use could be achieved by daily use for 121 days or could represent episodic use over several years.

Our exposure for the analyses of cognitive trajectory differed in a few ways from the one we defined for analyses of dementia. First, cumulative benzodiazepine use was calculated in the 10 years immediately before each study visit (that is, no one year lag) as we were evaluating cognitive decline only in participants who had not yet had a diagnosis of dementia. Second, we created a measure of recent use defined as filling two or more prescriptions for a benzodiazepine in the six months before each visit, requiring each prescription to have at least seven TSDDs (fig 2).

\section{Covariates}

Information about covariates came from standardized questionnaires administered at each study visit and from Group Health's electronic databases. Demographic factors included age, sex, and years of education. Body mass index (BMI) was determined from measured height and weight. ${ }^{29}$ Participants were asked about smoking, exercise, and self rated health. ${ }^{30}$ We ascertained presence of several comorbidities including hypertension or diabetes mellitus treated with drugs (computerized pharmacy data), history of stroke (self report or electronic databases), and coronary heart disease (self report). Symptoms of depression were obtained from the short version of the Center for Epidemiologic Studies Depression scale. ${ }^{31}$

\section{Statistical analyses}

\section{Analyses of dementia and Alzheimer's disease}

We used separate multivariable Cox proportional hazards models with participant's age as the time scale to estimate hazard ratios and $95 \%$ confidence intervals for the association between benzodiazepine use and incident dementia or possible or probable Alzheimer's disease. Age at study entry was taken as start of follow-up. Participants were followed until the earliest of onset of dementia, disenrollment from Group Health, or last study visit before 30 September 2012. We adjusted for age at study entry, sex, educational level, hypertension, diabetes mellitus, current smoking, stroke, coronary heart disease, BMI, regular exercise, self rated health, and symptoms of depression (variables defined in table 2). We included time varying measures for coronary heart

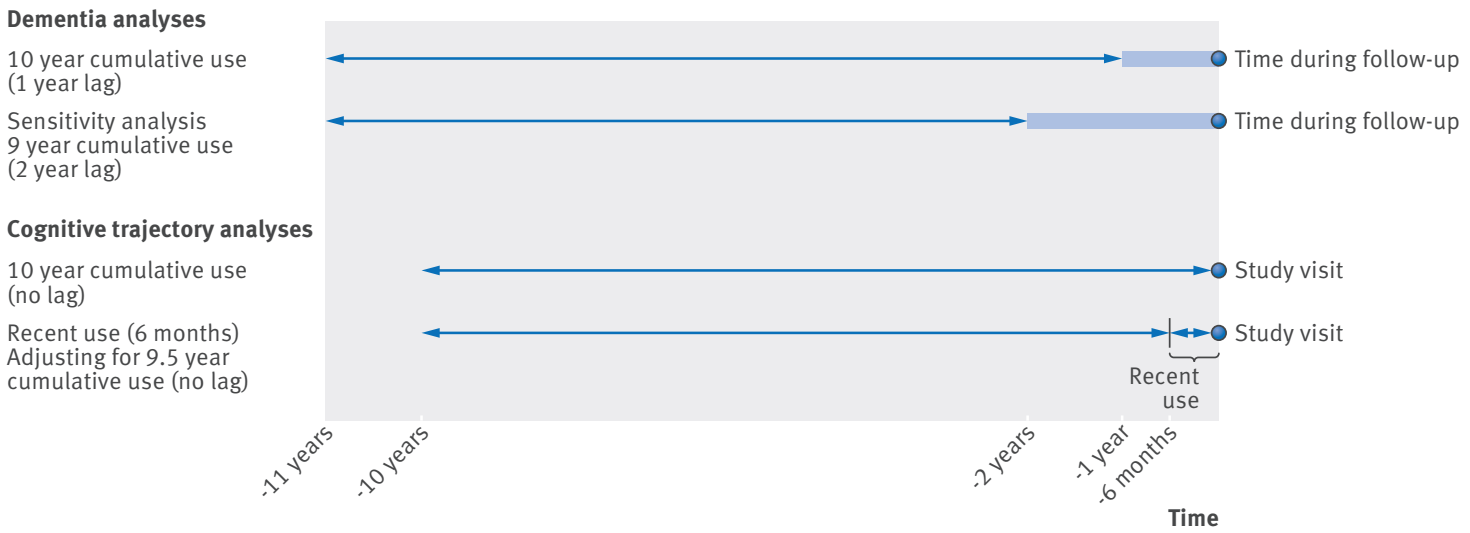

Fig 2 | Scheme for exposure definition for dementia and cognitive trajectory analyses. For analyses of dementia, a rolling 10 year window was used to define our time varying exposures. At each time point during follow-up, the 10 year cumulative exposure for all participants at risk is recalculated by summing all of their benzodiazepine use in the previous 10 years. The most recent year was excluded because of concerns about possible use for prodromal symptoms (shaded area). For analyses of cognitive trajectory, the circle at the far right represents a study visit at which the cognitive test was administered. Here, the year immediately before a study visit is not excluded from the cumulative use measure because by design, no participants could have a diagnosis of dementia at the time of a study visit included in these analyses. Recent exposure is defined as use in the six months immediately before the visit 


\begin{tabular}{|c|c|c|c|c|c|}
\hline \multirow[b]{2}{*}{ Baseline characteristics } & \multirow{2}{*}{$\begin{array}{l}\text { All participants } \\
(n=3434)\end{array}$} & \multicolumn{4}{|c|}{ Cumulative benzodiazepine use in 10 years before study entry (TSDD) } \\
\hline & & None $(n=2416)$ & $1-30(n=492)$ & $31-120(n=259)$ & $\geq 121(n=267)$ \\
\hline Median age (IQR) (years) & $74.4(70-80)$ & $74.4(70-80)$ & $74.2(70-79)$ & $74.4(70-79)$ & $75.1(70-80)$ \\
\hline Men & $1387(40.4)$ & $1050(43.5)$ & $156(31.7)$ & $94(36.3)$ & $87(32.6)$ \\
\hline Any college education & $2279(66.4)$ & $1589(65.8)$ & $336(68.3)$ & $178(69)$ & $176(65.9)$ \\
\hline Obese & $853(25.4)$ & $602(25.4)$ & $138(29.1)$ & $65(25.7)$ & $48(18.5)$ \\
\hline Current smoker & $173(5)$ & $122(5.1)$ & $27(5.5)$ & $12(4.7)$ & $12(4.5)$ \\
\hline Regular exerciset & $2453(71.6)$ & $1739(72.2)$ & $340(69.4)$ & $188(72.6)$ & $186(69.7)$ \\
\hline Fair or poor self rated health & $532(15.5)$ & $332(13.8)$ & $95(19.4)$ & $50(19.3)$ & $55(20.6)$ \\
\hline Treated hypertension $\neq$ & $1662(48.4)$ & $1109(45.9)$ & $263(53.5)$ & $144(55.6)$ & $146(54.7)$ \\
\hline Treated diabetes mellitis§ & $272(7.9)$ & $200(8.3)$ & $45(9.1)$ & $15(5.8)$ & $12(4.5)$ \\
\hline History of stroke & $221(6.4)$ & $125(5.2)$ & $49(10)$ & $25(9.7)$ & $22(8.2)$ \\
\hline Coronary heart disease** & $633(18.4)$ & $420(17.4)$ & $99(20.1)$ & $50(19.3)$ & $64(24)$ \\
\hline High depressive symptoms†† & $336(9.9)$ & $204(8.6)$ & $52(10.8)$ & $27(10.7)$ & $53(20)$ \\
\hline \multicolumn{6}{|c|}{ 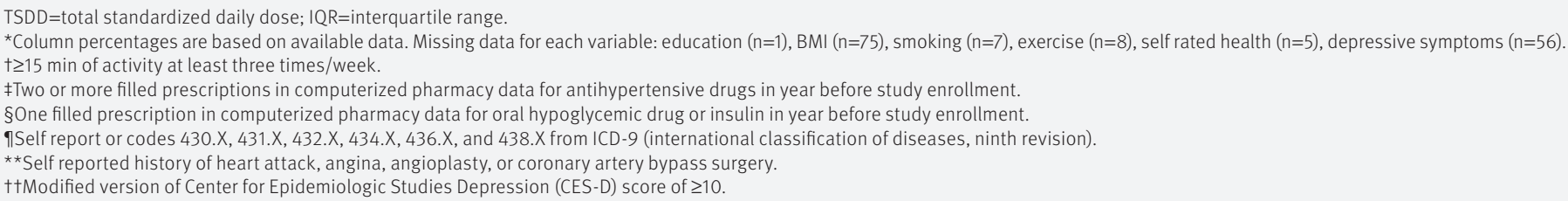 } \\
\hline
\end{tabular}

disease and stroke and the values from the baseline visit for all other covariates. We excluded observations with missing covariate information $(n=130)$. We assessed the assumption of proportional hazards by testing the interaction between the exposure and age at follow-up. In secondary analyses, we modeled the exposure as a continuous variable using natural cubic splines to examine whether results were influenced by the cutpoints chosen for exposure categories.

\section{Analyses of cognitive trajectory}

We evaluated the average differences in CASI scores and the average differences in rates of decline of these scores between benzodiazepine user groups using linear regression models estimated via generalized estimating equations. We used a working independence correlation matrix and calculated standard errors using the Huber-White sandwich estimator to account for the correlation between multiple CASI scores from the same individual. ${ }^{32}$ We estimated the average difference in rate of cognitive decline, defined as decline in CASI per year, between user groups by including an interaction term between age at follow-up and level of cumulative exposure. Models adjusted for the same covariates as in the dementia analyses. Analyses of the association between CASI trajectory and recent benzodiazepine use (six months before visit) also adjusted for cumulative use (six months to 10 years before visit).

\section{Sensitivity analyses}

For the dementia analyses, we performed several sensitivity analyses to explore the robustness of our results. Given the uncertainty regarding when prodromal symptoms might first emerge before a diagnosis of dementia, we extended the lag time to two years. In additional models, we included depressive symptoms as a time varying covariate and adjusted for the Charlson comorbidity index (Deyo adaption). ${ }^{33}$ Lastly, we performed a post hoc analysis extending the lag time to five years to replicate the methods used by another study. ${ }^{9}$

All analyses were performed with SAS version 9.2 (SAS Institute, Cary, NC).

\section{Patient involvement}

No patients were involved in setting the research question or the outcome measures, nor were they involved in recruitment or the design and implementation of the study. There are no plans to involve patients in the dissemination of results.

\section{Results}

Table 2 shows participants' characteristics overall and by cumulative benzodiazepine exposure in the 10 years before study entry. The median age of participants at study entry was $74,91 \%(n=3134)$ were white, $60 \%$ were women, and most (66\%) had some college education. Overall, 30\% had filled at least one prescription for a benzodiazepine in the 10 years before study entry, though lonely $3 \%(n=98)$ had recently used a benzodiazepine (within six months). Participants with heavier benzodiazepine use were more likely to be women and report fair or poor self rated health, have more depressive symptoms, and have comorbidities (such as hypertension, stroke, and coronary heart disease) than non-users. The most common benzodiazepines were temazepam, diazepam, clonazepam, triazolam, and lorazepam (table 3), which together accounted for 83\% of the benzodiazepine exposure. Within the highest benzodiazepine category ( $\geq 121$ TSDDs), the median level of use was 375 TSDDs (equivalent to slightly over a year of daily use).

\section{Dementia and Alzheimer's disease}

The 3434 participants included in these analyses accrued 25019 person years of follow-up, with a mean of 7.3 (SD 4.8) years. During this time, 797 (23.2\%) 


\begin{tabular}{|c|c|c|}
\hline Drug & $\begin{array}{l}\text { No }(\%) \text { of all participants } \\
(n=3434) \dagger\end{array}$ & $\begin{array}{l}\text { Total TSSD filled } \\
\text { (\% of total) }\end{array}$ \\
\hline Temazepam & $540(15.7)$ & $117349(21.8)$ \\
\hline Diazepam & $508(14.8)$ & $90085(16.7)$ \\
\hline Clonazepam & $77(2.2)$ & $88038(16.3)$ \\
\hline Triazolam & $265(7.7)$ & $76899(14.3)$ \\
\hline Lorazepam & $612(17.8)$ & 72405 (13.4) \\
\hline Alprazolam & $227(6.6)$ & $27765(5.2)$ \\
\hline Zolpidem & $126(3.7)$ & $25929(4.8)$ \\
\hline Flurazepam & $97(2.8)$ & $23994(4.5)$ \\
\hline Oxazepam & $85(2.5)$ & $12298(2.3)$ \\
\hline Chlordiazepoxide & $47(1.4)$ & $3097(0.6)$ \\
\hline Clorazepate & $5(0.2)$ & $1193(0.2)$ \\
\hline Eszopiclone & $3(0.1)$ & $159(0.0)$ \\
\hline Zaleplon & $2(0.1)$ & $62(0.0)$ \\
\hline Total & - & 539272 \\
\hline
\end{tabular}

TSDD=total standardized daily dose.

*Participant's study period included 10 years before study entry up to time of diagnosis of dementia or censored. TSDD summed for all participants for their entire study period. Zolpidem, eszopiclone, and zaleplon are non-benzodiazepine hypnotics that bind to same receptor as benzodiazepine drugs, hence are grouped together.

+Number of participants with at least 1 filled prescription for drug at any time during follow-up period. Participants could have prescriptions for multiple drugs so percentages do not sum to $100 \%$.

participants developed incident dementia, of whom 637 (79.9\%) developed Alzheimer's disease. Table 4 shows the follow-up time and number of events according to exposure categories. Figure 3 shows age adjusted and multivariable adjusted hazard ratios for dementia and Alzheimer's disease associated with cumulative benzodiazepine use. No association was found with the highest level of benzodiazepine use ( $\geq 121$ TSDDs) for dementia (hazard ratio 1.07, 95\% confidence interval 0.83 to 1.37 ) or Alzheimer's disease $(0.95,0.71$ to 1.27) compared with non-use. Relative to non-use, there was a slightly increased risk for dementia for participants with low (1-30 TSDDs; $1.25,1.03$ to 1.51 ) or moderate use (31-120 TSDDs; $1.31,1.00$ to 1.71 ); whereas for Alzheimer's disease, increased risk was noted only among participants with low use (1.27, 1.03 to 1.57). When we split the highest category of benzodiazepine use into two groups, the hazard ratio for dementia was 1.11 (0.78 to 1.58 ) for $121-364$ TSDDs and 1.03 (0.73 to 1.44 ) for $\geq 365$ TSDDs. Findings for Alzheimer's disease were similar.

Figure 4 shows results from additional analyses that modeled benzodiazepine exposure as a continuous variable with natural cubic splines. These curves show the estimated hazard ratios (and 95\% confidence intervals) for each level of exposure relative to a reference group with no cumulative exposure (0 TSDDs). For dementia, there was a modestly increased hazard ratio

\begin{tabular}{|c|c|c|c|}
\hline TSDD & $\begin{array}{l}\text { Follow-up time } \\
\text { (person years) }\end{array}$ & $\begin{array}{l}\text { Dementia } \\
\text { cases }\end{array}$ & $\begin{array}{l}\text { Alzheimer's } \\
\text { disease cases }\end{array}$ \\
\hline 0 & 16849 & 511 & 418 \\
\hline $1-30$ & 4099 & 148 & 120 \\
\hline $31-120$ & 1590 & 63 & 43 \\
\hline$\geq 121$ & 2481 & 75 & 56 \\
\hline
\end{tabular}

between 1.1 and 1.4 for $\leq 90$ TSDDs but risk declined toward 1.0 with higher exposure. Benzodiazepine use was not significantly associated with Alzheimer's disease at any exposure level.

When we extended the lag time to two years, the associations for the lowest level of benzodiazepine use were no longer significant for either dementia (hazard ratio 1.18, 95\% confidence interval 0.97 to 1.44 ) or Alzheimer's disease (1.18, 0.95 to1.47) (fig 3). Adjustment for depressive symptoms as a time varying covariate or for overall comorbidity did not alter estimates appreciably. In the post hoc analysis with a five year lag, we continued to find no association between cumulative benzodiazepine use and dementia (table 5).

\section{Cognitive trajectory}

The average CASI score at baseline was 93.4 (SD 4.7), with similar scores across levels of cumulative benzodiazepine use (range 93.0-93.6). We found no significant differences for any benzodiazepine use group in mean CASI or rates of decline compared with non-users (tables 6 and 7). Those in the highest benzodiazepine use group had a decline per year 0.002 points slower than people not using benzodiazepines (95\% confidence interval -0.05 to 0.06 ). There were also no differences in rates of cognitive decline between recent and non-recent users of benzodiazepines (adjusted coefficient $-0.0061,95 \%$ confidence interval -0.08 to 0.07 ). Results were similar when we used the CASI-IRT scores.

\section{Discussion}

In this population based longitudinal study of older adults with detailed pharmacy data and rigorous outcome assessment we did not find any association between the highest level of benzodiazepine use and dementia or cognitive decline. Contrary to expectations, we found a small increased risk for dementia in people with low (that is, up to one month) or moderate (one to four months) use. This pattern does not support a causal association between cumulative benzodiazepine use and risk of dementia, and the small increased risk observed with low use could represent treatment of prodromal symptoms, as supported by our sensitivity analyses. It is also possible that people with prodromal dementia, even years before diagnosis, could be more sensitive to benzodiazepine induced acute cognitive adverse events (for example, delirium), resulting in discontinuation of the drug and avoidance, in turn leading to low levels of use.

\section{Comparison with other studies}

Of the studies that used strategies to deal with reverse causation, our findings are in line with one recent study $^{14}$ but are in contrast with two previous studies that reported an increased risk of dementia with benzodiazepine use. ${ }^{89}$ In a case-control study conducted using administrative data from the UK based Clinical Practice Research Datalink, high use of benzodiazepines, as determined by number of prescriptions, was not associated with an increased risk of Alzheimer's disease after use initated in the prodromal phase was 


\section{Dementia}

1-30 TSDD

Age adjusted

Multivariable adjusted 1 year lag

Multivariable adjusted 2 year lag

31-120 TSDD

Age adjusted

Multivariable adjusted 1 year lag

Multivariable adjusted 2 year lag

$\geq 121$ TSDD

Age adjusted

Multivariable adjusted 1 year lag

Multivariable adjusted 2 year lag

Alzheimer's disease

1-30 TSDD

Age adjusted

Multivariable adjusted 1 year lag

Multivariable adjusted 2 year lag 31-120 TSDD

Age adjusted

Multivariable adjusted 1 year lag

Multivariable adjusted 2 year lag $\geq 121$ TSDD

Age adjusted

Multivariable adjusted 1 year lag

Multivariable adjusted 2 year lag

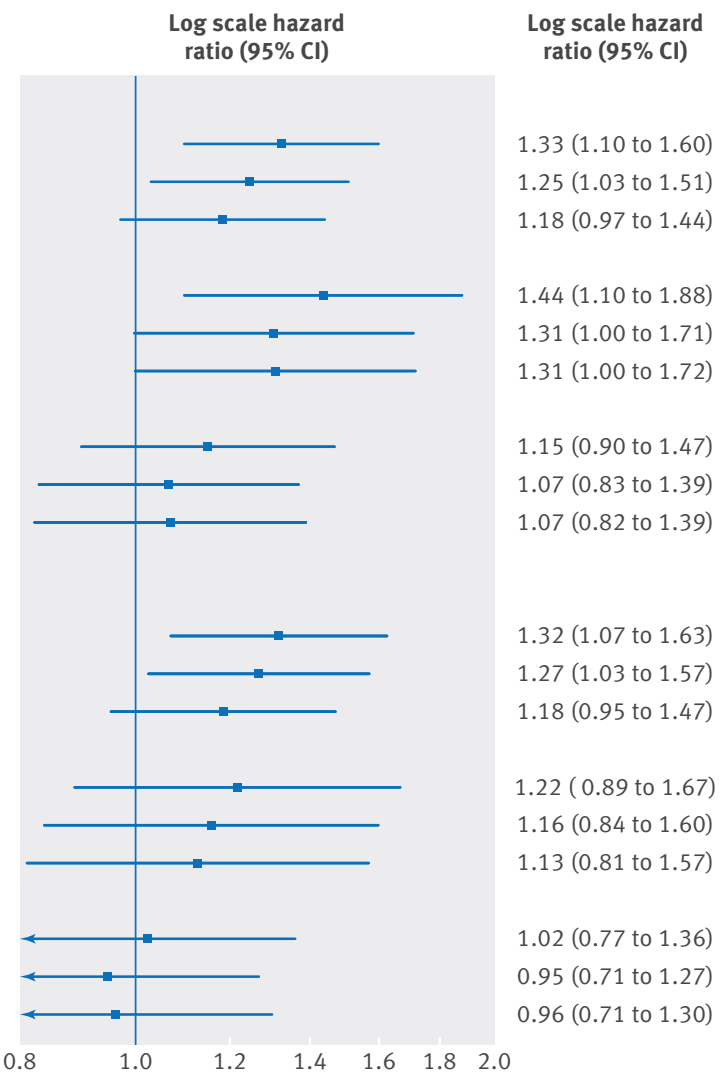

Fig 3 | Hazard ratios for all cause dementia and Alzheimer's disease for each level of cumulative benzodiazepine exposure compared with no use. Multivariable models adjusted for study cohort, age at study entry, sex, educational level, hypertension, diabetes mellitus, current smoking, stroke, coronary heart disease, BMI, regular exercise, self rated health, and depressive symptoms
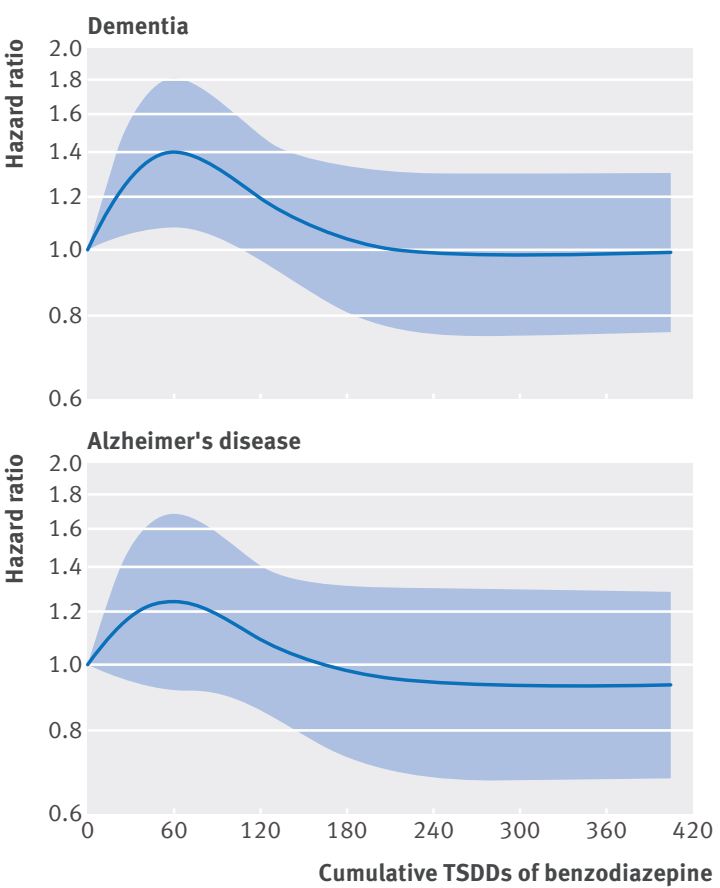

Fig 4 Association between cumulative benzodiazepine use modeled as spline and risk of incident dementia or Alzheimer's disease
Table 5 | Association between incident dementia and Alzheimer's disease and six year cumulative benzodiazepine use with five year lag time. *† Figures are hazard ratios $(95 \% \mathrm{Cl})$

\begin{tabular}{ll} 
TSDD & Adjusted model§ \\
Dementia & \\
\hline 0 & 1.00 (Reference) \\
\hline $1-30$ & $1.21(0.98$ to 1.50$)$ \\
\hline $31-120$ & $1.20(0.88$ to 1.64$)$ \\
\hline$\geq 121$ & $1.13(0.85$ to 1.52$)$ \\
\hline Alzheimer's disease & \\
\hline 0 & 1.00 (Reference) \\
\hline $1-30$ & $1.24(0.98$ to 1.57$)$ \\
\hline $31-120$ & 1.04 (0.72 to 1.51$)$ \\
\hline$\geq 121$ & 1.05 (0.75 to 1.46$)$ \\
\hline
\end{tabular}

TSDD=total standardized daily dose; ACT=Adult Changes in Thought.

* Observations with missing adjustment variables excluded from model ( $n=130 ; 3.8 \%$ )

tPrescriptions in five years before dementia onset excluded from calculation of exposure.

FTSDD example=minimum effective daily dose for temazepam is $15 \mathrm{mg}$ daily (=1 TSDD); person would fall into following TSDD category if they were using $15 \mathrm{mg}$ daily for 15 days (TSDD 1-30); $15 \mathrm{mg}$ daily for 90 days (TSDD 31-120); $15 \mathrm{mg}$ daily for 6 months (TSDD $\geq 121$ )

$\S$ Adjusted for ACT cohort, age (via the time-axis), age at ACT study entry, sex, educational level, BMI, current smoking, regular exercise, self-rated health, hypertension, diabetes mellitus, stroke, coronary heart disease, history of high depressive symptoms.

accounted for. In fact, people who filled more than 100 benzodiazepine prescriptions had a lower risk for Alzheimer's disease than non-users, a finding the authors cautioned against overinterpreting. ${ }^{14}$ In contrast, in a prospective population based study conducted in France of 1063 older adults, new use of benzodiazepines was associated with an increased risk of dementia (hazard ratio 1.62, 95\% confidence interval 1.08 to 2.43 ). ${ }^{8}$ In regard to this latter study, our results are not directly comparable because of differences in study design (their study included new users) and method of ascertaining benzodiazepine use (they relied on periodic interviews and lacked information about dose, duration, or chronicity of use). The study lacked information on some potential confounders and excluded $72 \%$ of the sample because of the new user design. In a case-control study among older adults living in Quebec, benzodiazepine use as assessed by computerized pharmacy data five to 10 years before the index date was associated with increased risk of Alzheimer's disease. ${ }^{9}$ These authors reported a dose-response relation with no association found for a cumulative dose of less than 91 prescribed daily doses but an increased risk for 91-180 prescribed daily doses $(1.32,1.01$ to 1.74$)$ and $>180$ prescribed daily doses $(1.84,1.62$ to 2.08$)$. After adjustment for diagnoses of depression, anxiety, and insomnia, estimated effects were slightly attenuated, and only the highest use category remained significantly associated with risk of Alzheimer's disease.

It is unclear why our findings differ from those of the Canadian study, but we offer a few possible explanations. Our participants could have had considerably lower use of benzodiazepines, although this is difficult to determine as that study did not report additional details about extent of exposure in their highest use category. If there is a true association, perhaps cumulative 


\begin{tabular}{|c|c|c|c|c|}
\hline TSDD & \multicolumn{2}{|l|}{ CASI } & \multicolumn{2}{|l|}{ CASI-IRT‡ } \\
\hline $1-30$ & $-0.16(-0.46$ to 0.14$)$ & $-0.11(-0.39$ to 0.16$)$ & $-0.01(-0.06$ to 0.03$)$ & $-0.02(-0.06$ to 0.02$)$ \\
\hline $31-120$ & $-0.08(-0.49$ to 0.34$)$ & $0.16(-0.22$ to 0.54$)$ & $0.01(-0.06$ to 0.07$)$ & $0.03(-0.03$ to 0.09$)$ \\
\hline \multicolumn{5}{|c|}{$\begin{array}{l}\text { TSDD=total standardized daily dose; CASI=cognitive abilities screening instrument; IRT=item response theory; ACT=Adult Changes in Thought. } \\
\text { *Linear regression with generalized estimating equations to account for repeated observations per participant. } \\
\text { †Negative values mean exposure category had lower mean CASI than reference group. For example, those in highest benzodiazepine group had mean } \\
\text { adjusted CASI score } 0.17 \text { points lower than non-user group. } \\
\text { †CASI-IRT had mean score of O (SD 1) among individuals without dementia at most recent study visit. } \\
\text { §Model adjusted for ACT cohort, age at study entry, sex, education, hypertension, diabetes, current smoking, stroke, coronary heart disease, BMI, } \\
\text { regular exercise, self rated health, and depressive symptoms. }\end{array}$} \\
\hline
\end{tabular}

use in our study fell below the threshold needed to increase risk of dementia. Although the method to calculate and categorize the cumulative daily dose differed in these studies, it is unlikely that these differences explain our discrepant results. Our analysis using continuous benzodiazepine exposure supported our primary findings of no association with higher doses. We explored extending the lag period used to exclude prescriptions because of prodromal symptoms to more closely match the Canadian study and continued to find no association. The primary difference between this study and ours relates to ascertainment of the outcome. The Canadian study relied on administrative data to identify cases of Alzheimer's disease; therefore, detection of Alzheimer's disease could be delayed compared with routine surveillance as used in our study, and there could be considerable misclassification of outcome status, which could be differential. People with heavy benzodiazepine use might have more frequent contact with the healthcare system, which could result in a higher likelihood of dementia being recognized and coded. While we cannot entirely rule out a moderate association in our highest benzodiazepine use group, the confidence limits exclude an association of the magnitude reported in the Canadian study.

Some of the challenges inherent to using an observational study design to examine benzodiazepine use and risk of dementia have been discussed, such as accounting for use of these drugs to treat prodromal symptoms and limitations to using administrative data for outcome ascertainment. Another methodological challenge is balancing the possible advantages of a new user design with the reality of how people use benzodiazepines (that is, potentially episodic use over several decades) and considerations of sample size. A new user design is one strategy to deal with the bias caused by depletion of susceptible users; however, it is not clear that this design is well suited to examine the principal hypothesis that heavier exposure (such as as might be accumulated over many years) is important for increasing risk of dementia. Such a design would be challenging to implement in association with prospective follow-up of a cohort with a rigorous process for ascertainment of dementia outcomes such as that used in our study, thus forcing the "new user" study to rely on a less optimal outcome definition such as diagnosis codes from administrative or automated data. Additionally, benzodiazepines might be used sporadically over long periods of time on an "as needed" basis, which could make it challenging to identify true "new users," particularly in elderly people. A prior study that attempted to identify new users in fact had limited data about benzodiazepine use before study enrollment, and so their apparent "new users" could in fact have had a history of benzodiazepine use, suggesting that even this study could have experienced bias because of depletion of susceptibles. ${ }^{8}$

\section{Strengths and limitations}

Our study has several strengths, including a large community based sample, an average follow-up of more than seven years, rigorous prospective ascertainment of dementia and Alzheimer's disease, and the ability to examine subclinical cognitive decline and dementia in the same cohort. We used computerized pharmacy data to characterize benzodiazepine use 10 years before

\begin{tabular}{|c|c|c|c|c|}
\hline \multirow[b]{2}{*}{ TSDD } & \multicolumn{2}{|l|}{ CASI } & \multicolumn{2}{|l|}{ CASI-IRT‡ } \\
\hline & Age adjusted( $95 \% \mathrm{CI})$ & $\begin{array}{l}\text { Multivariable adjusted } \\
(95 \% \mathrm{CI}) \S\end{array}$ & Age adjusted( $95 \% \mathrm{Cl})$ & $\begin{array}{l}\text { Multivariable adjusted } \\
(95 \% \mathrm{Cl}) \S\end{array}$ \\
\hline $1-30$ & $0.001(-0.04$ to 0.05$)$ & 0.006 (-0.04 to 0.05$)$ & $-0.0001(-0.01$ to 0.01$)$ & $0.0002(-0.01$ to 0.01$)$ \\
\hline $31-120$ & $0.032(-0.03$ to 0.09$)$ & $0.043(-0.01$ to 0.10$)$ & 0.0059 (0.00 to 0.01) & 0.0064 (0.00 to 0.01) \\
\hline
\end{tabular}


study entry and throughout follow-up, which, to our knowledge, is the longest duration used by any study to date for capturing benzodiazepine use. In addition, we were able to examine whether risk varies according to the extent of cumulative use. We specifically designed our study to take reverse causation into consideration and conducted multiple sensitivity and post hoc analyses to explore the impact of different choices related to defining exposure. Lastly, a further strength of this study is the covariate information collected directly from participants about characteristics that are not well measured in administrative data (such as physical activity; universal and standardized assessment of depression). A corresponding limitation, however, is that no data on these characteristics are available before study enrollment, and in some cases covariates were therefore assessed after the start of exposure. If these confounders lie in the causal pathway, this could result in overadjustment. For many of our covariates, we are not aware of evidence to suggest they might be consequences of (rather than predictors of) benzodiazepine use (such as hypertension, diabetes, coronary artery disease). In addition, our point estimates for the adjusted models were not considerably different than the age adjusted models, suggesting that the impact, if any, of such overadjustment was small.

A few limitations are worth mentioning. Few participants had heavy benzodiazepine use, and overall, our participants might have had lower levels of exposure than in some other studies. Like other studies that have examined benzodiazepine use and risk of dementia, we are unable to rule out depletion of susceptible bias. The non-user group might include individuals with past benzodiazepine use who had experienced acute cognitive adverse events because of underlying preclinical dementia pathology and therefore did not have the opportunity to accumulate higher benzodiazepine use. Other studies experience this limitation as well and therefore this potential bias does not explain the differences in study results. ${ }^{89}$ We were unable to exclude the possibility that within the source population, the most susceptible users of benzodiazepines might have developed dementia at a younger age and therefore been ineligible for study enrollment, which could have biased our findings toward not finding an association. We defined our exposure groups using a 10 year exposure window, and our highest group is likely to include heterogeneous exposure patterns (mix of chronic and intermittent users). Likewise, prior studies have not described or examined patterns of benzodiazpine use. ${ }^{89}$ Other designs would be necessary to try to address whether the specific pattern of benzodiazepine use is important for dementia risk.

The nature of the association between neuropsychiatric symptoms (such as depression, insomnia, and anxiety) and risk of dementia is unclear and might depend on the timing of the symptoms in relation to the diagnosis. These symptoms occurring in the years just before the diagnosis of dementia possibly represent prodromal symptoms, though these same symptoms occurring decades or more before the diagnosis might represent risk factors. ${ }^{34-36}$ Though we dealt with the possibility of benzodiazepine use for prodromal symptoms in our analysis, we were not able to adjust for anxiety and insomnia as risk factors. We did adjust for depressive symptoms, which are strongly correlated with anxiety. Results from a prior study suggest that adjustment for anxiety and insomnia are unlikely to considerably alter our findings, ${ }^{9}$ and, if anything, it would be expected to move our hazard ratio closer toward the null. We were unable to ascertain whether prescribers had discontinued benzodiazepines because they identified that a participant had cognitive changes (such as delirium) while taking these drugs, which might have limited our ability to examine benzodiazepine use and cognitive trajectories. Furthermore, the CASI is a screening tool, making it relatively insensitive to detect cognitive decline in people with high cognitive functioning. However, we still did not find an association between benzodiazepine use and cognitive decline when we used methods to overcome this limitation by using the transformed CASI scores. Lastly, as most participants were white and relatively well educated, our results might not be generalizable to other groups.

\section{Clinical implications and conclusions}

In conclusion, we found a slightly higher risk of dementia in people with the lowest benzodiazepine use but no increased risk in those with the highest level of exposure (median exposure equivalent to about one year of daily use). Overall, our pattern of findings does not support the theory that cumulative benzodiazepine use at the levels observed in our population is causally related to an increased risk for dementia or cognitive decline. It should be noted that our study did not examine the acute cognitive adverse events that can occur when treatment with a benzodiazepine is started in older adults and careful monitoring is recommended in this situation. Nonetheless, given the mixed evidence regarding benzodiazepines and risk of dementia and that these drugs are associated with many adverse events, healthcare providers are still advised to avoid benzodiazepines in older adults to prevent important adverse health outcomes, withdrawal, and dependence.

We thank Susan McCurry, Wayne McCormick, and James Bowen, who participated in multidisciplinary consensus committee meetings that determined study participants' dementia status. This paper was presented at the 20th Annual HMO Research Network Conference in Phoenix, AZ, on 3 April 2014.

Contributors: SLG, MA, SD, RW, RAH, OY, and EBL contributed to study conception and design; all authors contributed to acquisition, analysis, or interpretation of data; SLG and OY drafted the manuscript; all authors revised the manuscript for critical intellectual content; OY conducted statistical analyses; and SD, EBL, and PKC obtained funding. All authors had full access to all of the data (including statistical reports and tables) in the study and take responsibility for the integrity of the data and the accuracy of the data analysis. SLG and $\mathrm{EBL}$ are guarantors.

Funding: This work was supported by National Institute on Aging ( $\mathrm{NIH}$ Grants U01AG00678 (EBL), R03AG042930 (SD)) and by the Branta Foundation (SD). The sponsors had no role in design and conduct of the study; collection, management, analysis, and interpretation of the data; or in preparation, review, or approval of the manuscript; or the decision to submit the manuscript for publication. 
Competing interests: All authors have completed the Unified Competing Interest form at www.icmje.org/coi_disclosure.pdf (available on request from the corresponding author) and declare that SD received a Merck/American Geriatrics Society New Investigator Award; EB receives royalties from UpToDate; RW received funding as a biostatistician from a research grant awarded to Group Health Research Institute from Pfizer; OY received funding as a biostatistician from research grants awarded to Group Health Research Institute from Amgen and Bayer.

Ethical approval: The research protocol for this study was approved by the institutional review boards of Group Health and University of Washington. Written informed consent was obtained from all participants.

Transparency declaration: SLG affirms that the manuscript is an honest, accurate, and transparent account of the study being reported; that no important aspects of the study have been omitted; and that any discrepancies from the study as planned (and, if relevant, registered) have been explained.

Data sharing: No additional data available.

This is an Open Access article distributed in accordance with the Creative Commons Attribution Non Commercial (CC BY-NC 3.0) license, which permits others to distribute, remix, adapt, build upon this work non-commercially, and license their derivative works on different terms, provided the original work is properly cited and the use is noncommercial. See: http://creativecommons.org/licenses/by-nc/3.0/.

1 Olfson M, King M, Schoenbaum M. Benzodiazepine use in the United States. JAMA Psychiatry 2015;72:136-42. doi:10.1001/ jamapsychiatry.2014.1763

2 Koyama A, Steinman M, Ensrud K, Hillier TA, Yaffe K. Ten-year trajectory of potentially inappropriate medications in very old women: importance of cognitive status. J Am Geriatr Soc 2013;61:258-63. doi:10.1111/jgs.12093.

3 Pisani MA, Murphy TE, Araujo KL, Slattum P, Van Ness PH, Inouye SK. Benzodiazepine and opioid use and the duration of intensive care unit delirium in an older population. Crit Care Med 2009;37:177-83. doi:10.1097/CCM.0b013e318192fcf9.

4 American Geriatrics Society 2012 Beers Criteria Update Expert Panel. American Geriatrics Society updated Beers Criteria for potentially inappropriate medication use in older adults. J Am Geriatr Soc 2012;60:616-31. doi:10.1111/j.1532-5415.2012.03923.x

5 Tannenbaum C, Paquette A, Hilmer S, Holroyd-Leduc|, Carnahan R. A systematic review of amnestic and non-amnestic mild cognitive impairment induced by anticholinergic, antihistamine, GABAergic and opioid drugs. Drugs Aging 2012;29:639-58.

6 Mura T, Proust-Lima C, Akbaraly T, et al. Chronic use of benzodiazepines and latent cognitive decline in the elderly: results from the Three-city study. Eur Neuropsychopharmacol 2013;23:21223. doi:10.1016/i.euroneuro.2012.05.004

7 Verdoux H, Lagnaoui R, Begaud B. Is benzodiazepine use a risk facto for cognitive decline and dementia? A literature review of epidemiological studies. Psychol Med 2005;35:307-15. doi:10.1017/ S0033291704003897.

8 Billioti de Gage S, Bégaud B, Bazin F, et al. Benzodiazepine use and risk of dementia: prospective population based study. BMJ 2012;345:e6231. doi:10.1136/bmj.e6231.

9 Billioti de Gage S, Moride Y, Ducruet T, et al. Benzodiazepine use and risk of Alzheimer's disease: case-control study. BMJ 2014;349:g5205. doi:10.1136/bmi.g5205.

10 Lagnaoui R, Bégaud B, Moore N, et al. Benzodiazepine use and risk of dementia: a nested case-control study. J Clin Epidemiol 2002;55:3148. doi:10.1016/S0895-4356(01)00453-X

11 Wu CS, Ting TT, Wang SC, Chang IS, Lin KM. Effect of benzodiazepine discontinuation on dementia risk. Am / Geriatr Psychiatry 2011;19:151-9. doi:10.1097/JGP.0b013e3181e049ca.

12 Wu CS, Wang SC, Chang IS, Lin KM. The association between dementia and long-term use of benzodiazepine in the elderly: nested case-control study using claims data. Am J Geriatr Psychiatry 2009;17:614-20. doi:10.1097/JGP.0b013e3181a65210.

13 Gallacher J, Elwood P, Pickering J, Bayer A, Fish M, Ben-Shlomo Y. Benzodiazepine use and risk of dementia: evidence from the Caerphilly Prospective Study (CaPS). J Epidemiol Community Health 2012;66:869-73. doi:10.1136/jech-2011-200314.

14 Imfeld P, Bodmer M, Jick SS, Meier CR. Benzodiazepine Use and risk of developing Alzheimer's Disease or vascular dementia: A case-control analysis. Drug Saf 2015;38:909-19. doi:10.1007/s40264-015-0319-3.
15 Amieva H, Le Goff M, Millet X, et al. Prodromal Alzheimer's disease: successive emergence of the clinical symptoms. Ann Neurol 2008:64:492-8. doi:10.1002/ana.21509.

16 Stella F, Radanovic M, Balthazar ML, Canineu PR, de Souza LC, Forlenza OV. Neuropsychiatric symptoms in the prodromal stages of dementia. Curr Opin Psychiatry 2014;27:230-5. doi:10.1097/YCO.0000000000000050.

17 Richard E, Reitz C, Honig LH, et al. Late-life depression, mild cognitive impairment, and dementia. JAMA Neurol 2013;70:374-82.

18 Kukull WA, Higdon R, Bowen JD, et al. Dementia and Alzheimer disease incidence: a prospective cohort study. Arch Neurol 2002;59:1737-46. doi:10.1001/archneur.59.11.1737.

19 Teng EL, Hasegawa K, Homma A, et al. The Cognitive Abilities Screening Instrument (CASI): a practical test for cross-cultural epidemiological studies of dementia. Int Psychogeriatr 1994;6:45-58, discussion 62. doi:10.1017/S1041610294001602.

20 Diagnostic and Statistical Manual of Mental Disorders.4th ed. American Psychiatric Association, 1994.

21 McKhann G, Drachman D, Folstein M, Katzman R, Price D, Stadlan EM. Clinical diagnosis of Alzheimer's disease: report of the NINCDS ADRDA Work Group under the auspices of Department of Health and Human Services Task Force on Alzheimer's Disease. Neurology 1984:34:939-44. doi:10.1212/WNL.34.7.939.

22 Crane PK, Narasimhalu K, Gibbons LE, et al. Item response theory facilitated cocalibrating cognitive tests and reduced bias in estimated rates of decline. J Clin Epidemiol 2008;61:1018-27.e9. doi:10.1016/j. jclinepi.2007.11.011.

23 Ehlenbach WJ, Hough CL, Crane PK, et al. Association between acute care and critical illness hospitalization and cognitive function in older adults. JAMA 2010;303:763-70. doi:10.1001/jama.2010.167.

24 Semla TP, Beizer JL, Higbee MD. Geriatric Dosage Handbook.15th ed. Lexicomp, 2010

25 Gray SL, Anderson ML, Dublin S, et al. Cumulative use of strong anticholinergics and incident dementia: a prospective cohort study. JAMA Intern Med 2015;175:401-7. doi:10.1001/ jamainternmed.2014.7663.

26 Gray SL, LaCroix AZ, Blough D, Wagner EH, Koepsell TD, Buchner D. Is the use of benzodiazepines associated with incident disability?] Am Geriatr Soc 2002;50:1012-8. doi:10.1046/j.1532-5415.2002.50254.x.

27 Hanlon JT, Boudreau RM, Roumani YF, et al. Number and dosage of central nervous system medications on recurrent falls in community elders: the Health, Aging and Body Composition study. Jerontol A Biol Sci Med Sci 2009;64:492-8. doi:10.1093/gerona/ gln043.

28 Tamim H, Monfared AA, LeLorier J. Application of lag-time into exposure definitions to control for protopathic bias. Pharmacoepidemiol Drug Saf 2007:16:250-8. doi:10.1002/ pds.1360.

29 Clinical Guidelines on the Identification, Evaluation, and Treatment of Overweight and Obesity in Adults. 2014. http://www.nhlbi.nih.gov/ files/docs/guidelines/obesity_guidelines_archive.pdf

30 Larson EB, Wang L, Bowen JD, et al. Exercise is associated with reduced risk for incident dementia among persons 65 years of age and older. Ann Intern Med 2006:144:73-81. doi:10.7326/0003-4819-144-2-200601170-00004.

31 Andresen EM, Malmgren JA, Carter WB, Patrick DL. Screening for depression in well older adults: evaluation of a short form of the CES-D (Center for Epidemiologic Studies Depression Scale). Am J Prev Med 1994;10:77-84

32 Zeger SL, Liang KY. Longitudinal data analysis for discrete and continuous outcomes. Biometrics 1986:42:121-30. doi: $10.2307 / 2531248$.

33 Deyo RA, Cherkin DC, Ciol MA. Adapting a clinical comorbidity index for use with ICD-9-CM administrative databases. / Clin Epidemiol 1992;45:613-9. doi:10.1016/0895-4356(92)90133-8.

34 Johansson L, Guo X, Waern M, et al. Midlife psychological stress and risk of dementia: a 35-year longitudinal population study. Brain 2010:133:2217-24. doi:10.1093/brain/awq116.

35 Skogen JC, Bergh S, Stewart R, Knudsen AK, Bjerkeset O. Midlife mental distress and risk for dementia up to 27 years later: the Nord-Trøndelag Health Study (HUNT) in linkage with a dementia registry in Norway. BMC Geriatr 2015;15:23. doi:10.1186/ s12877-015-0020-5.

36 Spira AP, Chen-Edinboro LP, Wu MN, Yaffe K. Impact of sleep on the risk of cognitive decline and dementia. Curr Opin Psychiatry 2014;27:478-83. doi:10.1097/YCO.0000000000000106. 